\title{
Materials Science of Vision Correction: Glasses and Sunglasses
}

Glass lenses have been used to improve eyesight since at least the 13th century when Roger Bacon, an English philosopher and scientist, mentioned their use in a manuscript written in 1267. Concave lenses provide correction for nearsightedness, while convex lenses correct farsightedness.

By the 17th century, opticians in London had developed an iron tool with convex and concave sides for grinding and polishing lenses of optical (clear) glass. A glass "blank" was glued temporarily to the convex side, then covered with an abrasive liquid such as emery and water and ground down by hand with the concave side. Final polishing was carried out with the concave side covered by a wool cloth and a powder of calcined tin. But lenses prepared by this technique were of uneven quality and had to be produced one at a time by skilled opticians. The Worshipful Company of Spectacle Makers, founded in London in 1629 , was a guild to which most opticians belonged and set standards for the quality of lenses.

John Marshall (?1659-1725), who served as optician to King George I, revolutionized the manufacture of spectacles by developing an improved grinding process that could produce uniformly ground lenses in batches. Instead of using optical glass, he used "crystal-glass," also known as agate, a form of quartz with small grain size. Agate lenses proved to be harder than those made of optical glass. Marshall fashioned his grinding tools from brass rather than iron because brass could take a smoother polish and in turn produce higher quality polished lenses. The new tools held four blanks that could be ground simultaneously (still by hand) to identical focal length. As a result of these improvements, spectacles both increased in quality and became more widely available at lower cost. By the 19th century, a lathe was used to turn the grinding tool. Though many additional improvements have followed, the batch method is still in use in the modern era.

Until the 18th century, spectacles were held to the face by a spring-loaded grip on the bridge of the nose. In French, spectacles are still known as "pince-nez," or pinch-nose. Alternatively, lenses were sometimes mounted in a frame that could be held up to the eyes when needed. Around 1725, British optician Edward Scarlatt invented temples, the hinged portion of modern eyeglasses that rest over the ears.

Many people find they need different

lenses for near and distant focus, particularly as they reach middle age. In the late 1700 s, Benjamin Franklin became frustrated by his frequent need to switch glasses and tried slicing his reading lenses and distance lenses in half, then gluing half of each reading lens below half of each distance lens. The result was the first pair of bifocals. Further development took place early in the 20th century, when John Borsch, Jr. cut a segment out of a distance lens and filled it with a segment of a reading lens, then heated the two sections to join them together. Later it became possible to grind a single lens with one focal length for the top half and another for the bottom half. All of these methods left obvious lines through the lenses that were unattractive and distracting to the wearer. Trifocals were invented in the 1940s, but the subtle head-tilt needed to make use of the different parts of the lenses was difficult to master. By the 1950s, "progressive addition lenses" were invented in France by Bernard Maitenaz. These lenses provide a continuous gradation in corrective power without sharp transitions from one part of the lens to another and remain popular today, both for their ease of use and their cosmetic appeal.

The Inuit (Eskimo) and Copper Culture peoples have traditionally used "sunglasses" or snow goggles made from wood, walrus tusk, bone, or hide.

Until the late 1950s, all corrective lenses were made of glass. Since then, various kinds of plastic have been in regular use. Polycarbonate plastic lenses came into use in the mid-1980s. Plastic's main advantage over glass is its lower density, requiring only half (or even less) the weight of glass for the same prescription. It is also much safer than glass, being more fracture resistant and less likely to chip at an exposed edge. Glass has the advantage of blocking ultraviolet (uv) light, and is extremely scratch resistant. Special coatings on plastic lenses can provide uv filtering and improve scratchresistance. Anti-reflective coatings allow the wearer's eyes to be visible, and are based on the method developed in the late 1930 s by Katherine Blodgett, who worked with Irving Langmuir at General Electric. (Her technique for making antireflective coatings was also used to make periscopes, range finders, and aerial cameras for use in World War II.)

The Inuit (Eskimo) and Copper Culture peoples have traditionally used "sunglasses" or snow goggles made from wood, walrus tusk, bone, or hide. These have no glass but the eye slits are narrow enough to protect the eyes from the glare of sun reflected from ice and snow. Modern sunglasses were originally just tinted glasses. Tinting can be incorporated into the lens itself or added as a coating. Cheap plastic lenses without uv filtering can actually harm eyes: By blocking visible light, they allow the iris to remain relatively open, leaving the eye extra vulnerable to uv damage. Polarizing lenses were introduced in sunglasses because of their usefulness in absorbing the glare of reflected light.

Photochromatic (or photochromic) lenses change from transparent to dark in sunlight. Early work in this area was carried out by S. Donald Stookey and other scientists at Corning Glass Works in the 1940s. Crystallites of copper-doped silver halide are distributed through a glass matrix. Incident uv light excites electrons from the copper atoms; the electrons are absorbed by silver ions, which become neutral and aggregate to form small clusters that absorb visible light, darkening the lens. Early models required at least a minute to change color but more recently developed technologies have a much faster response time, a necessity for the driver encountering a quick transition from bright sunlight to a darkened tunnel. Difficulties remain, though: Photochromatic lenses do not always turn as dark as regular sunglasses, and often do not change color well inside a car where uv intensity may not be sufficient to trigger the photochromatic dye. Research on improved photochromatic lenses continues with some emphasis on the use of high molecular weight liquid crystals.

ROBIN L. BLUMBERG SELINGER

FOR FURTHER READING: Jeffrey Anshel, Healthy Eyes Better Vision (Los Angeles, The Body Press, 1990); and the University of Waterloo's Museum of Visual Science and Optometry at http: / / quark.uwaterloo.ca $/ \sim$ museum $/$. See D.J. Bryden and D.L. Sims, British Medical Journal 309 (1994) p. 1713 for more information on John Marshall. 